\title{
Quark-Hadron Model for Elastic Scattering Between Two-Composite Systems at Relativistic Energies
}

\author{
M.M.H. El-Gogary \\ Physics Department, Faculty of Science, Cairo University, Giza, Egypt
}

\begin{tabular}{|c|c|}
\hline ARTICLE INFO & ABSTRACT \\
\hline Article history: & \multirow{9}{*}{$\begin{array}{l}\text { The author of the present study has previously developed formulas to calculate the full } \\
\text { Glauber series of the elastic scattering between two composite systems. Their structures } \\
\text { are pictured as a bag of quarks or as a cluster of nucleons. In this work, a hybrid model in } \\
\text { which the quark-hadron pictures are combined was constructed. The elastic cross sections } \\
\text { of alpha-proton at center of mass energy } \sqrt{\mathrm{s}}=89 \mathrm{GeV} \text { and alpha-alpha at } \sqrt{\mathrm{s}}=126 \mathrm{GeV} \\
\text { are evaluated by the hybrid model, the pure quark and conventional nucleon models. The } \\
\text { results of different approaches are compared with the experimental data. The comparison } \\
\text { shows that the predictions of the three model are identical at forward scattering angles and } \\
\text { are significantly different at large momentum transfers. At large angles, the hybrid model } \\
\text { calculation fits very well the experimental data when compared the calculations of quark } \\
\text { and nucleon models. The nucleon model prediction provides a better agreement with the } \\
\text { data than the quark model calculation. The improvement achieved by the hybrid model } \\
\text { over the quark model, at large scattering angles, shows that the inclusion of quark-quark } \\
\text { interactions is more consistent when the separation between the colliding nucleons is less } \\
\text { than the hadron core. }\end{array}$} \\
\hline Received: $22^{\text {nd }}$ Feb. 2021 & \\
\hline Accepted: $8^{\text {th }}$ Aug. 2021 & \\
\hline Keywords: & \\
\hline Elastic cross-section, & \\
\hline $\begin{array}{l}\text { Glauber multiple scattering } \\
\text { theory, }\end{array}$ & \\
\hline Quark-hadron model, & \\
\hline Alpha scattering, & \\
\hline Proton scattering. & \\
\hline
\end{tabular}

\section{INTRODUCTION}

Elastic scattering between two composite particles at high energies is always one of the powerful tools to investigate the structure of their constituents. The experimental results of such interactions showed that the angular distributions measured at small scattering angles exhibit the trend of diffraction pattern resulting from the multiple scattering between the constituents of composite particles [1-13]. Furthermore, at large momentum transfers $(q)$, the differential cross sections decay to values many orders of magnitude smaller than those in the forward direction [14-17]. Moreover, with the aid of the present-day accelerators such collisions are performed at ultra-relativistic energies and their cross sections are measured over wider ranges of momentum transfers [1828]. The recent data are also having the same characteristics but with various degrees depending on the values of incident energy and constituent number of the colliding systems. Surely, data available with such details are very useful to test more rigorously the theoretical models concerned and their ranges of validity.
As for the situation concerning the theories, different phenomenological models have been developed to study the elastic cross sections of such experimental data. Indeed, until this moment no model manages to describe qualitatively and quantitatively the large amount of data exist; they all have merits and shortcomings. Typically, they successfully describe the experimental results in a certain kinematic range, but completely fail in other ones.

The Glauber multiple scattering theory [29-31] is one of these models which has been developed to describe such collisions at high-energies. Although Adachi and Kotani [32-37] and Islam [38, 39] have derived more admissible formulas of the elastic scattering amplitude accounting as well for region of $q$ values delivered at finite collision energies, still the Glauber high energy formula of the scattering amplitude are extensively applied. In fact, the Glauber analysis is more attractive because the scattering amplitude representing the collision between two composite particles is simply expressed by the multiple scattering between their constituents. Second, practically speaking, this model 
can obtain a microscopic description for these reactions even if one uses simple uncorrelated wave functions and effective elemental scattering amplitudes. Indeed, the theory has shown great success in describing the elastic scattering data of hadron-nucleus interactions. The data are excellently reproduced especially when the higher orders of the multiple scattering series are considered [40-43]. For nucleus-nucleus scattering, the earlier analyses made by this theory have shown disagreements with the experimental data at small angles and/or unphysical divergence at large momentum transfers [4143]. However, the authors of these calculations attributed this failure to the simplifying approximations made. They truncated all higher orders of the multiple scattering series, a matter which omits the effective role of such orders at large scattering angels. Furthermore, the effect of the center-of-mass correlation is treated as a global correction factor for all orders of multiple scattering, a matter which destroy the translation invariance symmetry of the scattering amplitude. Moreover, all the nucleus-nucleus reactions applied have medium bombarding energies, and such energies neither sufficiently high to use Glauber model nor appropriate to employ the single Gaussian parametrization for NN scattering amplitude $[41,42]$. Later, an attempt truncated the multiple scattering series showed that the drawback of the large $q$ divergence is removed by incorporating the center-ofmass correlations in the phase shift function with the same order as the scattering terms [43]. Clearly, without avoiding these drawbacks, the predictive power of Glauber approach cannot be assessed for analyzing composite particle scattering.

As a matter of fact, the multiple scattering series of Glauber contains $\left(2^{A \times B}-1\right)$ terms so that its complete summation is very extensive and computationally difficult for collisions with mass numbers $A, B \geq 4$. Furthermore, the model calculations involve multidimensional integrals, which are cumbersome to be evaluated, even if one uses simple Gaussian forms for the nuclear densities and NN scattering amplitudes. Unfortunately, at a high energy the momentum transfer of the composite particle scattering is significantly large, so the c. m. correlations should be treated in a consistent manner and the higher orders of the multiple scattering series are necessary to enhance the predictions at large angles.

Owing to these difficulties, Yin et al. [44,50] with aid of the theory of permutation group have introduced a method to classify the multiple scattering terms into sets, each set contains the terms of equal contribution to the scattering amplitude. As a result, these terms of equal contribution are represented by one typical term, named 'orbit', and their number is referred to as the length of the corresponding orbit. The Gaussian forms for the nuclear densities and NN scattering amplitudes have transformed the multiple integral of each orbit into simple algebraic recurrence formulas. However, it has been proven that the classification method is practically useful for calculating the full Glauber series of the multiple scattering between systems whose mass numbers $\leq 4$ [47-50]. Later on, El-Gogary et al. have corrected the cluster approach proposed by Huang [51] to extend the application of Yin method to classify the multiple scattering terms of heavier systems [52,53] and treated the center-of-mass correlations of such systems in a consistent manner [54]. Although, the formulas developed in such attempts are a practical approximation to the exact Glauber series calculation, but their applications show unsatisfactory descriptions for elastic collisions at medium energies. However, such failure was attributed to the inaccurate values of the $N N$ parameters used at medium energies and the approximation of the $N N$ amplitude by simple Gaussian form generally correct at a high energy.

For analyzing composite scatterings at very high energies, many investigations reported that the precise description of Glauber multiple scattering theory requires the consideration of quarks as a substructure inside the nucleon [55-58]. The first Glauber model calculation taking this consideration was made by Franco [55] to investigate $\alpha-p$ and $\alpha-\alpha$ elastic scattering at center of mass energy $\sqrt{\mathrm{s}}=89 \mathrm{GeV}$ and $126 \mathrm{GeV}$, respectively. In this attempt, the author has truncated the multiple scattering series between quarks of such systems up to fifth order scattering terms and parameterized the quark-quark ( $q q)$ scattering amplitudes and the quark densities with single Gaussian forms. The $q q$ parameters needed in these calculations are obtained by fitting the $N N$ elastic scattering data. Unfortunately, these truncated Glauber series calculation of quark model underestimated the full series evaluation of the conventional nucleonic picture. Subsequent studies, approximated also the calculation of full Glauber series, have been performed to investigate the same reactions with what is called the hybrid quark-hadron $(\mathrm{HQH})$ model [56,57]. The model is hybrid because it switches on the $q q$ interactions of the nuclear systems just for collision with impact parameter $b \leq b_{o} \mathrm{fm}, b_{o}$ which is taken to be the radius of the hadron, and describes the collision of $b>b_{o} \mathrm{fm}$ outside the hadronic region with the conventional $N N$ interactions. The calculations are performed by taking the same forms previously reported [55] for $q q$ and $N N$ 
scattering amplitudes, but with more suitable parameters using HQH model. The elastic angular distribution of $\alpha-p$ scattering obtained using the $\mathrm{HQH}$ picture has shown a better agreement with the experimental data than the previous calculations, especially at large momentum transfer [56]. For $\alpha-\alpha$ scattering, the situation is not the same. The agreement is somewhat enhanced, but the prediction of $\mathrm{HQH}$ model to the scattering data for this case is clearly not satisfactory at large angles. The truncation of the higher order multiple scattering terms made in Glauber series becomes serious for this case.

Following the remarkable analyses made by other authors [55-57], El-Gogary et al. shared these efforts with an attempt other investigators [58] in which the quark and nucleon models are constructed using the formulas previously developed. [52-54]. We thought at that time that if one takes the full Glauber series of the multiple scattering between quarks in the composite systems, and utilizes reliable parameterizations of quark densities and $N N$ scattering amplitudes such as the single Gaussian forms which are more accurate at relativistic energies, this may make the quark model more realistic than the nucleon one at such energies. However, the calculation of $\alpha-p$ scattering performed in this attempt shows an insignificant improvement, leading us to stop any further calculations to $\alpha-\alpha$ scattering.

In the present study, the author tends to improve the previously reported analysis [58] by constructing a hybrid model in which the quark and hadron models of the scattering between two composite systems were combined using the full Glauber series calculations. This combination is carried out by configurating the space of the impact parameter (b) of the two-composite scattering amplitude into two distinct regions: the interior quark region $\left(b \leq b_{o}\right)$ and the exterior hadronic region $\quad(b>$ $b_{o}$ ) where $b_{o}=0.7 \mathrm{fm}$, which is taken to be the boundary separating the two regions. This would include the contribution of quark degrees of freedom more consistently in composite scattering at relativistic energies. For evaluation, the full Glauber multiple scattering series calculation of the hybrid model was performed and the results obtained are compared with the quark and nucleonic models [58] calculated independently. The formulas of the developed hybrid model presented here are given in section (2). The results of the differential cross sections of $\alpha-p$ and $\alpha-\alpha$ interactions using this study and their discussion are given in section (3). Section (4) is left to the conclusion. The orbits, lengths and $\Delta$-matrices required for the calculations are exhibited in the Appendix I.

\section{THEORY}

In this section, a suitable approach is developed to determine the elastic scattering between two composite nuclear systems of nucleon numbers $m$ and $n$ at relativistic energies. A hybrid quark-hadron model combining between two structures of the colliding systems was developed, namely the nucleon model as $m$ and $n$ nucleons and the quark model as $3 m$ and $3 n$ quarks, and involving the full Glauber series describing the multiple scattering between the constituents of each picture. According to this hybrid model, the whole elastic scattering amplitude of projectile $A$ and target $\quad B$ combining the two pictures may be written as follows[56]:

$$
F_{A B}(\vec{q})=F_{Q Q}^{b \leq b_{o}}(\vec{q})+F_{N N}^{b>b_{o}}(\vec{q})
$$

$F_{Q Q}^{b \leq b_{o}}(\vec{q})$, stands for the scattering amplitude of two composite systems of $3 m$ and $3 n$ quarks in the inner quark region of impact parameter $b \leq b_{o}$ can be written in Glauber model as:

$F_{Q Q}^{b \leq b_{o}}(\vec{q})=\frac{i k}{2 \pi} \Theta(\vec{q}) \int_{0}^{b_{o}} d \vec{b} \exp (i \vec{q} \cdot \vec{b})\left\{1-\exp \left[i \chi_{Q Q}(\vec{b})\right]\right\}$

$F_{N N}^{b>b_{o}}(\vec{q})$, stands for the scattering amplitude of two composite systems of $m$ and $n$ nucleons in the outer hadronic region of impact parameter $b>b_{o}$, which can similarly be given as:

$F_{N N}^{b>b_{o}}(\vec{q})=\frac{i k}{2 \pi} \Theta(\vec{q}) \int_{b_{O}}^{\infty} d \vec{b} \exp (i \vec{q} \cdot \vec{b})\left\{1-\exp \left[i \chi_{N N}(\vec{b})\right]\right\}$ (3)

Where, $\vec{q}$ is the momentum transferred from the projectile A to the target B. $\Theta(\vec{q})$ is the global correction arising from the effect of the center-of-mass correlations [42], $\vec{k}$ is the incident momentum of the projectile, $\vec{b}$ is the impact parameter vector and $b_{o}$ is a phenomenological parameter. $\chi_{Q Q}(\vec{b})$ and $\chi_{N N}(\vec{b})$ are the nuclear phase shift functions representing the scattering of two composite systems as bags of quarks and nucleons, respectively. The modified scattering amplitude $\bar{F}(\vec{q})$ involving the center-of-mass correlations consistently not as a global correction factor $\Theta(\vec{q})$ as in Eq. 2 or 3 but with the same order as the phaseshift expansion has a general form

$\bar{F}(\vec{q})=\frac{i k}{2 \pi} \int d \vec{b} \exp (i \vec{q} \cdot \vec{b})\{1-\exp [i \bar{\chi}(\vec{b})]\}$

$\bar{\chi}(\vec{b})$, the modified nuclear phase shift function containing the center-of-mass correlations, is related to the uncorrelated one, $\chi(\vec{b})$ by $[41,42]$

$\exp [i \bar{\chi}(\vec{b})]=\int_{0}^{\infty} J_{o}(q b) \Theta(\vec{q}) q d q \int_{0}^{\infty} J_{o}\left(q b^{\prime}\right) \exp \left[i \chi\left(\vec{b}^{\prime}\right)\right] b^{\prime} d b^{\prime}$

Using the additivity assumption in Glauber approximation [41], the total phase shift function $\chi_{A B}$ of the colliding system 
can be expressed in terms of the elemental phase shifts $\chi_{i j}$ of their constituents as:

$\chi_{A B}\left(\vec{b},\left\{\vec{s}_{i}\right\},\left\{\vec{s}_{j}^{\prime}\right\}\right)=\sum_{i=1}^{A} \sum_{j=1}^{B} \chi_{i j}\left(\vec{b}+\vec{s}_{i}-\vec{s}_{j}^{\prime}\right)$

with form $\chi_{Q Q}\left(\vec{b},\left\{\vec{s}_{i}\right\},\left\{\vec{s}_{j}^{\prime}\right\}\right)=\sum_{i=1}^{A=3 m} \sum_{j=1}^{B=3 n} \chi_{i j}\left(\vec{b}+\vec{s}_{i}-\vec{s}_{j}^{\prime}\right)$ for quark model

or

with form $\chi_{N N}\left(\vec{b},\left\{\vec{s}_{i}\right\},\left\{\vec{s}_{j}^{\prime}\right\}\right)=\sum_{i=1}^{A=m} \sum_{j=1}^{B=n} \chi_{i j}\left(\vec{b}+\vec{s}_{i}-\vec{s}_{j}^{\prime}\right)$ for hadronic model

and both are generally given by:

$\exp \left[i \chi_{A B}(\vec{b})\right]=\left\langle\Psi_{A}\left(\left\{\vec{r}_{i}\right\}\right) \Psi_{B}\left(\left\{\vec{r}_{j}^{\prime}\right\}\right)\left|\exp \left[i \chi_{A B}\left(\vec{b},\left\{\vec{s}_{i}\right\},\left\{\vec{s}_{j}^{\prime}\right\}\right)\right]\right| \Psi_{A} \Psi_{B}\right\rangle(\mathbf{7})$

Where, $\Psi_{A}\left(\left\{\vec{r}_{i}\right\}\right)\left[\Psi_{B}\left(\left\{\vec{r}_{j}^{\prime}\right\}\right)\right]$ is the ground state wave function of the projectile [target] system, $\left\{\vec{s}_{i}\right\}\left[\left\{\vec{s}_{j}^{\prime}\right\}\right]$ is the projection of the relative position vector $\left\{\vec{r}_{i}\right\}\left[\left\{\vec{r}_{j}^{\prime}\right\}\right]$ of the projectile [target] particles on the impact parameter plane. With the definition of the profile function,

$\Gamma_{A B}(\vec{b})=1-\exp \left[i \chi_{A B}(\vec{b})\right]$, the phase shift operator takes the following form:

$$
\exp \left[i \chi_{A B}\left(\vec{b},\left\{\vec{s}_{i}\right\},\left\{\vec{s}_{j}^{\prime}\right\}\right)\right]=\prod_{i=1}^{A} \prod_{j=1}^{B}\left[1-\Gamma_{i j}\left(\vec{b}+\vec{s}_{i}-\vec{s}_{j}^{\prime}\right)\right]
$$

Where, $\Gamma_{i j}\left(\vec{b}+\vec{s}_{i}-\vec{s}_{j}^{\prime}\right)$ represents the profile function of the two-particle scattering. Expanding the products in Eq. (6) gives the Glauber series representing the multiple scattering between the constituents of the composite system. The number of terms contained in such series is $\left(2^{A \times B}-1\right)$ terms, each term represents the multiple scattering with specific order. For practical calculations, the approach employed by El-Gogary [54] was followed. In this approach, the cluster structure is considered for the colliding systems. Suppose there are $\mathrm{M}_{\mathrm{A}}$ clusters in system $\mathrm{A}$ and $\mathrm{M}_{\mathrm{B}}$ clusters in system $B$, and there are $M_{N}$ particles in each cluster $\left(M_{N}\right.$ is a common divisor for $\mathrm{A}$ and $\mathrm{B}$ ) under this treatment, Eq. (6) is reexpressed as:

$\exp \left[i \chi_{A B}\left(\vec{b},\left\{\vec{s}_{i \alpha}\right\},\left\{\vec{s}_{j \delta}^{\prime}\right\}\right)\right]=\prod_{i=1}^{M_{A}} \prod_{j=1}^{M_{B}}\left[1-\Gamma_{i j}\left(\vec{b},\left\{\vec{s}_{i \alpha}\right\},\left\{\vec{s}_{j \delta}^{\prime}\right\}\right)\right]$ (9)

with

$\Gamma_{i j}\left(\vec{b},\left\{\vec{s}_{i \alpha}\right\},\left\{\vec{s}_{j \delta}^{\prime}\right\}\right)=1-\prod_{\alpha=1}^{M_{N}} \prod_{\delta=1}^{M_{N}}\left[1-\Gamma_{i \alpha, j \delta}\left(\vec{b}+\vec{s}_{i \alpha}-\vec{s}_{j \delta}^{\prime}\right)\right]$

Where $\Gamma_{i j}$ represents the profile function of scattering between the ith cluster in projectile $\mathrm{A}$ and jth cluster in target $\mathrm{B}$ and $\Gamma_{i \alpha, j \delta}$ is the scattering between the $\alpha$ th particle of the ith cluster in projectile $A$ and $\delta$ th particle of jth cluster in target B.

Adopting Yin et al. method [44], the multiple scattering terms of Eq. (9) can be classified into sets; each set contains the terms of equal contribution to the scattering amplitude. All terms in each set are represented by one typical term, referred to as orbit, and the number of terms in this set is referred to as the length of that orbit. Having utilized this classification, the nuclear phase shift takes the form:

$\exp \left[i \chi_{A B}\left(\vec{b},\left\{\vec{s}_{i \alpha}\right\},\left\{\vec{s}_{j \delta}^{\prime}\right\}\right)\right]=1+\sum_{V_{1}=1}^{M_{1}} \sum_{\lambda_{V_{1}}} T_{1}\left(V_{1}, \lambda_{V_{1}}\right) \prod_{i=1}^{M_{A}} \prod_{j=1}^{M_{B}}$

$$
\begin{array}{r}
{\left[\sum _ { V _ { 2 } = 1 } ^ { M _ { 2 } } \sum _ { \lambda _ { V _ { 2 } } } T _ { 2 } ( V _ { 2 } , \lambda _ { V _ { 2 } } ) \prod _ { \alpha = 1 } ^ { M _ { N } } \prod _ { \delta = 1 } ^ { M _ { N } } \left[-\Gamma_{i \alpha, j \delta}\left(\vec{b}+\vec{s}_{i \alpha}-\right.\right.\right.} \\
\left.\left.\left.\vec{s}_{j \delta}^{\prime}\right)\right]^{\Delta_{i \alpha, j \delta}\left(V_{2}, \lambda_{V_{2}}\right)}\right]^{\Delta_{i j}\left(V_{1}, \lambda_{V_{1}}\right)}
\end{array}
$$

Where, $M_{1}=M_{A} \times M_{B}, M_{2}=M_{N} \times M_{N}$, and the indices $\left(V, \lambda_{V}\right)$ characterize an orbit with order of scattering $V$ and serial index $\lambda_{V}$. Each orbit $\left(V_{1}, \lambda_{V_{1}}\right)\left[\left(V_{2}, \lambda_{V_{2}}\right)\right]$ is represented by an $M_{A} \times M_{B}\left[M_{N} \times M_{N}\right]$ dimensional matrix whose elements $\Delta_{i j}\left(V_{1}, \lambda_{V_{1}}\right)\left[\Delta_{i \alpha, j \delta}\left(V_{2}, \lambda_{V_{2}}\right)\right]$ have only two values one, if $\Gamma_{i j}\left[\Gamma_{i \alpha, j \delta}\right]$ appears in the expansion term and zero if it is absent. $T_{1}\left(V_{1}, \lambda_{V_{1}}\right)$ $\left[T_{2}\left(V_{2}, \lambda_{V_{2}}\right)\right]$ is the length of the orbit $\left(V_{1}, \lambda_{V_{1}}\right)\left[\left(V_{2}, \lambda_{V_{2}}\right)\right]$ which is determined by using the properties of the permutation group, $G_{1}=S_{M_{A}} \otimes S_{M_{B}}$ $\left[G_{2}=S_{M_{N}} \otimes S_{M_{N}}\right]$. The scattering amplitude $f_{i \alpha, j \delta}(\vec{q})$ is related to the profile function $\Gamma_{i \alpha, j \delta}(\vec{b})$ by:

$\Gamma_{i \alpha, j \delta}(\vec{b})=\frac{1}{2 \pi i k_{N}} \int d \vec{q} \exp (-\vec{q} \cdot \vec{b}) f_{i \alpha, j \delta}(\vec{q})$

Assuming, for simplicity, that all the two-particle amplitudes are equal, (which is approximately true at a high energy) $f_{i \alpha, j \delta}(\vec{q})$ can be parameterized by $[55,56]$ :

$f_{i \alpha, j \delta}(\vec{q})=\frac{k_{N} \sigma}{4 \pi}(i+\varepsilon) \exp \left(-\mu q^{2} / 2\right)$

Where, $k_{N}$ is the momentum of the incident particle, $\sigma$, is the total cross-section and $\varepsilon$, is the ratio of the real to the imaginary parts of the forward scattering amplitude. $\mu$ is the slope parameter of the elastic scattering differential cross-section. Inserting Eq. (13) into Eq. (12) and doing the integration, we get

$$
\begin{gathered}
; \Gamma_{i \alpha, j \delta}\left(\vec{b}+\vec{s}_{i \alpha}-\vec{s}_{j \delta}^{\prime}\right)=g \exp \left[-\left(\vec{b}+\vec{s}_{i \alpha}-\vec{s}_{j \delta}^{\prime}\right)^{2} / 2 \mu\right] \\
\text { where, } g=\frac{\sigma}{4 \pi \mu}(1-i \varepsilon)
\end{gathered}
$$

Substituting with Eq. (14) into Eq. (11), we get: 


$$
\begin{aligned}
\exp \left[i \chi_{A B}\left(\vec{b},\left\{\vec{s}_{i \alpha}\right\},\left\{\vec{s}_{j \delta}^{\prime}\right\}\right)\right]=1+\sum_{V_{1}=1}^{M_{1}} \sum_{\lambda_{V_{1}}} T_{1}\left(V_{1}, \lambda_{V_{1}}\right) \\
\prod_{i=1}^{M_{A}} \prod_{j=1}^{M_{B}} \\
\left\{\sum _ { V _ { 2 } = 1 } ^ { M _ { 2 } } \sum _ { \lambda _ { V _ { 2 } } } ( - g ) ^ { V _ { 2 } } T _ { 2 } ( V _ { 2 } , \lambda _ { V _ { 2 } } ) \operatorname { e x p } \left[-\frac{1}{2 \mu} \sum_{\alpha=1}^{M_{N}} \sum_{\delta=1}^{M_{N}}(\vec{b}+\right.\right. \\
\left.\left.\left.\vec{s}_{i \alpha}-\vec{s}_{j \delta}^{\prime}\right)^{2} \Delta_{i \alpha, j \delta}\left(V_{2}, \lambda_{V_{2}}\right)\right]\right\}^{\Delta_{i j}\left(V_{1}, \lambda_{V_{1}}\right)}
\end{aligned}
$$

Where $\quad V_{2}=\sum_{\alpha=1}^{M_{N}} \sum_{\delta=1}^{M_{N}} \Delta_{i \alpha, j \delta}\left(V_{2}, \lambda_{V_{2}}\right)$.

The form of wave function of the system is needed to perform the integration of Eq. (7). Considering the approximation in which the particles inside any cluster and the clusters themselves inside the system are completely uncorrelated, then it could be written as follows:

$$
\begin{aligned}
& \left|\Psi_{A}\left(\left\{\vec{r}_{i \alpha}\right\}\right) \Psi_{B}\left(\left\{\vec{r}_{j \delta}^{\prime}\right\}\right)\right|^{2}= \\
& \quad\left[\prod_{i=1}^{M_{A}} \prod_{\alpha=1}^{M_{N}} \rho_{A}\left(\vec{r}_{i \alpha}\right)\right]\left[\prod_{j=1}^{M_{B}} \prod_{\delta=1}^{M_{N}} \rho_{B}\left(\vec{r}_{j \delta}^{\prime}\right)\right]
\end{aligned}
$$

Where $\rho_{A}\left(\vec{r}_{i \alpha}\right)$ and $\rho_{B}\left(\vec{r}_{j \delta}^{\prime}\right)$ are the normalized single particle density functions and are chosen to be the singleGaussian form:

$$
\rho_{\gamma}(\vec{r})=\left(E_{\gamma}\right)^{3 / 2} \exp \left(-\eta_{\gamma}{ }^{2} r^{2}\right), \gamma=A, B
$$

Where, $E_{\gamma}=\eta_{\gamma}{ }^{2} / \pi$ and $\eta_{\gamma}{ }^{2}$ is a size parameter related to the mean square radius $\left\langle r_{\gamma}{ }^{2}\right\rangle$ measured for the nucleus. Because the incident direction of the projectile $\mathrm{A}$ is chosen to be the z-axis, $\chi_{A B}(\vec{b})$ has no dependence on such variable in Glauber approximation, the integration of the nuclear densities over the variables $z_{i \alpha}$ and $z_{j \delta}^{\prime}$ in Eq. (7) is performed directly and the remaining is the transverse densities given as:

$\left|\Psi_{A}\left(\left\{\vec{s}_{i \alpha}\right\}\right) \Psi_{B}\left(\left\{\vec{s}_{j \delta}^{\prime}\right\}\right)\right|^{2}=$

$$
\left\{\prod_{i=1}^{M_{A}} c_{A} \exp \left[-\sum_{\alpha=1}^{M_{N}} \eta_{A}^{2} s_{i \alpha}^{2}\right]\right\}\left\{\prod_{j=1}^{M_{B}} c_{B} \exp \left[-\sum_{\delta=1}^{M_{N}} \eta_{B}^{2} s_{j \delta}^{\prime 2}\right]\right\}
$$

Where, $c_{A}=\left(E_{A}\right)^{M_{N}} \quad, \quad c_{B}=\left(E_{B}\right)^{M_{N}}$

With the phase shift function (15), the density (18), and the differential element

$$
\begin{aligned}
d \tau= & {\left[\prod_{i=1}^{M_{A}} \prod_{\alpha=1}^{M_{N}} d s_{i \alpha}\right]\left[\prod_{j=1}^{M_{B}} \prod_{\delta=1}^{M_{N}} d s_{j \delta}^{\prime}\right] } \\
& =\left[\prod_{i=1}^{M_{A}} \prod_{\alpha=1}^{M_{N}} d x_{i \alpha} d y_{i \alpha}\right]\left[\prod_{j=1}^{M_{B}} \prod_{\delta=1}^{M_{N}} d x_{j \delta}^{\prime} d y_{j \delta}^{\prime}\right]
\end{aligned}
$$

Eq. (7) becomes, $\exp \left[i \chi_{A B}(\vec{b})\right]=1+$

$\sum_{V_{1}=1}^{M_{1}} \sum_{\lambda_{V_{1}}} T_{1}\left(V_{1}, \lambda_{V_{1}}\right) \prod_{i=1}^{M_{A}} \prod_{j=1}^{M_{B}}\left[\boldsymbol{c}_{A} \boldsymbol{c}_{\boldsymbol{B}} *\right.$

$\sum_{V_{2}=1}^{M_{2}} \sum_{\lambda_{V_{2}}}(-g)^{V_{2}} T_{2}\left(V_{2}, \lambda_{V_{2}}\right) \int\left(\prod_{\alpha=1}^{M_{N}} d s_{i \alpha}\right)\left(\prod_{\delta=1}^{M_{N}} d s_{j \delta}^{\prime}\right) \exp -\left\{\sum_{\alpha=1}^{M_{N}} \eta_{A}^{2} s_{i \alpha}^{2}-\right.$

$\sum_{\delta=1}^{M_{N}} \eta_{B^{2}}^{2}{ }_{j \delta}^{2}-\frac{1}{2 \mu} \sum_{\alpha=1}^{M_{N}} \sum_{\delta=1}^{M_{N}}\left(\vec{b}+\vec{s}_{i \alpha}-\right.$

$\left.\left.\left.\vec{s}_{j \delta}^{\prime}\right)^{2} \Delta_{i \alpha, j \delta}\left(V_{2}, \lambda_{V_{2}}\right)\right\}\right]^{\Delta_{i j}\left(V_{1}, \lambda_{V_{1}}\right)}$
Eq. (20) can be written as:

$$
\begin{array}{r}
\exp \left[i \chi_{A B}(\vec{b})\right]=1+\sum_{V_{1}=1}^{M_{1}} \sum_{\lambda_{V_{1}}} T_{1}\left(V_{1}, \lambda_{V_{1}}\right) \prod_{i=1}^{M_{A}} \prod_{j=1}^{M_{B}}\left[\boldsymbol{c}_{A} \boldsymbol{c}_{\boldsymbol{B}}\right. \\
\left.* \sum_{V_{2}=1}^{M_{2}} \sum_{\lambda_{V_{2}}} T_{2}\left(V_{2}, \lambda_{V_{2}}\right)(-g)^{V_{2}} J_{\left(V_{2}, \lambda_{V_{2}}, \Delta_{i \alpha, j \delta}\left(V_{2}, \lambda_{V_{2}}\right)\right)}(\vec{b})\right]^{\Delta_{i j}\left(V_{1}, \lambda_{V_{1}}\right)}
\end{array}
$$

Where,

$$
\begin{aligned}
& J_{\left(V_{2}, \lambda_{V_{2}}, \Delta_{i \alpha, j \delta}\left(V_{2}, \lambda_{V_{2}}\right)\right)}(\vec{b})= \\
& \int\left(\prod_{\alpha=1}^{M_{N}} d s_{i \alpha}\right)\left(\prod_{\delta=1}^{M_{N}} d s_{j \delta}^{\prime}\right) \exp \left\{-\sum_{\alpha=1}^{M_{N}} \eta_{A}^{2} s_{i \alpha}^{2}\right. \\
& \quad-\sum_{\delta=1}^{M_{N}} \eta_{B}^{2} s_{j \delta}^{\prime 2}-\frac{1}{2 \mu} \sum_{\alpha=1}^{M_{N}} \sum_{\delta=1}^{M_{N}}\left(\vec{b}+\vec{s}_{i \alpha}-\right. \\
& \left.\left.\vec{s}_{j \delta}^{\prime}\right)^{2} \Delta_{i \alpha, j \delta}\left(V_{2}, \lambda_{V_{2}}\right)\right\}
\end{aligned}
$$

The multiple integral in Eq. (22) can be solved recursively yielding the result:

$$
\begin{aligned}
& J_{\left(V_{2}, \lambda_{V_{2}}, \Delta_{i \alpha, j \delta}\left(V_{2}, \lambda_{V_{2}}\right)\right)}(\vec{b})= \\
& R\left[V_{2}, \lambda_{V_{2}}, \Delta_{i \alpha, j \delta}\left(V_{2}, \lambda_{V_{2}}\right)\right] \exp \left[-S\left(V_{2}, \lambda_{V_{2}}, \Delta_{i \alpha, j \delta}\left(V_{2}, \lambda_{V_{2}}\right)\right) b^{2}\right]
\end{aligned}
$$

With $R\left(V_{2}, \lambda_{V_{2}}, \Delta_{i \alpha, j \delta}\left(V_{2}, \lambda_{V_{2}}\right)\right)=$ $\left[\prod_{\alpha=1}^{M_{N}}\left(4 \pi \mu^{2} T_{\alpha}\left(i, j, V_{2}, \lambda_{V_{2}}\right)\right)\right]\left[\prod_{\delta=1}^{M_{N}}\left(\frac{\pi}{a_{\delta \delta}(i, j, \delta)}\right)\right]$ and, $S\left(V_{2}, \lambda_{V_{2}}, \Delta_{i \alpha, j \delta}\left(V_{2}, \lambda_{V_{2}}\right)\right)=a_{o}(\delta)-\sum_{\delta=1}^{M_{N}} \frac{c_{\delta}^{2}(\delta)}{4 a_{\delta \delta}(i, j, \delta)}$

Where the symbols appeared are defined as:

$$
\begin{aligned}
& \sigma_{\delta}\left(i, j, V_{2}, \lambda_{V_{2}}\right)=\sum_{\alpha=1}^{M_{N}} \Delta_{i \alpha, j \delta}\left(V_{2}, \lambda_{V_{2}}\right) \\
& \rho_{\alpha}\left(i, j, V_{2}, \lambda_{V_{2}}\right)=\sum_{\delta=1}^{M_{N}} \Delta_{i \alpha, j \delta}\left(V_{2}, \lambda_{V_{2}}\right) \\
& T_{\alpha}\left(i, j, V_{2}, \lambda_{V_{2}}\right)=1 / 2 \mu\left(2 \mu \eta_{A}^{2}+\rho_{\alpha}\left(i, j, V_{2}, \lambda_{V_{2}}\right)\right)
\end{aligned}
$$

and the coefficients $a$ 's and $c$ 's are determined using the simple algebraic recursion formulas:

$$
\begin{aligned}
& a_{o}\left(M_{N}\right)=\sum_{\delta=1}^{M_{N}} \eta_{B}^{2} \\
& a_{\delta \delta}\left(i, j, M_{N}\right)=\eta_{B}^{2}+\frac{1}{2 \mu} \sigma_{\delta}\left(i, j, V_{2}, \lambda_{V_{2}}\right)- \\
& \sum_{\alpha=1}^{M_{N}} T_{\alpha}\left(i, j, V_{2}, \lambda_{V_{2}}\right) \Delta_{i \alpha, j \delta}\left(V_{2}, \lambda_{V_{2}}\right) \\
& a_{\delta \beta}\left(i, j, M_{N}\right)= \\
& 2 \sum_{\alpha=1}^{M_{N}} T_{\alpha}\left(i, j, V_{2}, \lambda_{V_{2}}\right) \Delta_{i \alpha, j \delta}\left(V_{2}, \lambda_{V_{2}}\right) \Delta_{i \alpha, j \beta}\left(V_{2}, \lambda_{V_{2}}\right) \\
& c_{\delta}\left(M_{N}\right)=2 \eta_{B}^{2} \\
& a_{\delta \delta}\left(i, j, M_{N}-1\right)=a_{\delta \delta}\left(i, j, M_{N}\right)-\frac{a_{\delta M_{N}}^{2}\left(i, j, M_{N}\right)}{4 a_{M_{N} M_{N}\left(i, j, M_{N}\right)}}
\end{aligned}
$$




$$
\begin{aligned}
& a_{\delta \beta}\left(i, j, M_{N}-1\right)=a_{\delta \beta}\left(i, j, M_{N}\right)+ \\
& \frac{a_{\delta M_{N}}\left(i, j, M_{N}\right) a_{\beta M_{N}}\left(i, j, M_{N}\right)}{2 a_{M_{N}} M_{N}\left(i, j, M_{N}\right)} \quad, \quad \beta>\delta \\
& c_{\delta}\left(M_{N}-1\right)=c_{\delta}\left(M_{N}\right)+\frac{c_{M_{N}}\left(M_{N}\right) a_{\delta M_{N}}\left(i, j, M_{N}\right)}{2 a_{M_{N} M_{N}}\left(i, j, M_{N}\right)}
\end{aligned}
$$

The details of the integration method are given in previous study [54]. Thus, the following result could be obtained:

$$
\begin{aligned}
& \exp \left[i \chi_{A B}(\vec{b})\right]=1+\sum_{V_{1}=1}^{M_{1}} \sum_{\lambda_{V_{1}}} T_{1}\left(V_{1}, \lambda_{V_{1}}\right) \prod_{i=1}^{M_{A}} \prod_{j=1}^{M_{B}}\left[c_{A} c_{B}\right. \\
& \sum_{V_{2}=1}^{M_{2}} \sum_{\lambda_{V_{2}}}(-g)^{V_{2}} * T_{2}\left(V_{2}, \lambda_{V_{2}}\right) R\left(V_{2}, \lambda_{V_{2}}, \Delta_{i \alpha, j \delta}\left(V_{2}, \lambda_{V_{2}}\right)\right) \\
& \left.\exp \left\{-S\left(V_{2}, \lambda_{V_{2}}, \Delta_{i \alpha, j \delta}\left(V_{2}, \lambda_{V_{2}}\right)\right) b^{2}\right\}\right]^{\Delta_{i j}\left(V_{1}, \lambda_{V_{1}}\right)}
\end{aligned}
$$

For incorporating the center of mass correlations in the phase shift function, let us write $\exp \left[i \chi_{A B}(\vec{b})\right]$, Eq. (26) , as:

$$
\begin{aligned}
& \exp \left[i \chi_{A B}(\vec{b})\right]=1+ \\
& \sum_{V_{1}=1}^{M_{1}} \sum_{\lambda_{V_{1}}} T_{1}\left(V_{1}, \lambda_{V_{1}}\right) \prod_{i=1}^{M_{A}} \prod_{j=1}^{M_{B}}[Z]^{\Delta_{i j}\left(V_{1}, \lambda_{V_{1}}\right)}
\end{aligned}
$$

Where,

$$
\begin{aligned}
& Z=\boldsymbol{c}_{\boldsymbol{A}} \boldsymbol{c}_{\boldsymbol{B}} \sum_{V_{2}=1}^{M_{2}} \sum_{\lambda_{V_{2}}}(-g)^{V_{2}} T_{2}\left(V_{2}, \lambda_{V_{2}}\right) R\left(V_{2}, \lambda_{V_{2}}, \Delta_{i \alpha, j \delta}\left(V_{2}, \lambda_{V_{2}}\right)\right) \\
& \quad * \exp \left[-S\left(V_{2}, \lambda_{V_{2}}, \Delta_{i \alpha, j \delta}\left(V_{2}, \lambda_{V_{2}}\right)\right) b^{2}\right]
\end{aligned}
$$

In addition, the center of mass correction factor $\Theta(\vec{q})=$ $\exp \left[\frac{q^{2}}{4}\left(\frac{1}{A \eta_{A}^{2}}+\frac{1}{B \eta_{B}^{2}}\right)\right]$ [42] would be:

$$
\Theta_{Q Q}(\vec{q})=\exp \left[\frac{q^{2}}{4}\left(\frac{1}{A \eta_{A}^{2}}+\frac{1}{B \eta_{B}^{2}}\right)\right] \text { with } A=3 m, B=3 n
$$

(the correction for quark model)

Or

$$
\Theta_{N N}(\vec{q})=\exp \left[\frac{q^{2}}{4}\left(\frac{1}{A \eta_{A}^{2}}+\frac{1}{B \eta_{B}^{2}}\right)\right] \text { with } A=m, B=n
$$

(the correction for hadronic model)

The modified phase shift function $\bar{\chi}_{A B}(\vec{b})$ can be similarly written as:

$\exp \left[i \bar{\chi}_{A B}(\vec{b})\right]=1+$

$\sum_{V_{1}=1}^{M_{1}} \sum_{\lambda_{V_{1}}} T_{1}\left(V_{1}, \lambda_{V_{1}}\right) \prod_{i=1}^{M_{A}} \prod_{j=1}^{M_{B}}[\bar{Z}]^{\Delta_{i j}\left(V_{1}, \lambda_{V_{1}}\right)}$

Inserting the expression of $Z$, Eq. (28), and the center of mass correction, into Eq. (5),

$\bar{Z}$ is obtained by the form:

$$
\begin{array}{ll}
\bar{Z}=c_{A} c_{B} \sum_{V_{2}=1}^{M_{2}} \sum_{\lambda_{V_{2}}} T_{2}\left(V_{2}, \lambda_{V_{2}}\right)(-g)^{V_{2}} & \bar{R}\left(V_{2}, \lambda_{V_{2}}, \Delta_{i \alpha, j \delta}\left(V_{2}, \lambda_{V_{2}}\right)\right) \\
\quad * \exp \left\{-\bar{S}\left[V_{2}, \lambda_{V_{2}}, \Delta_{i \alpha, j \delta}\left(V_{2}, \lambda_{V_{2}}\right)\right] b^{2}\right\} & \text { (30) } \\
\text { with, } \quad \bar{S}=\left[\frac{1}{S}-\frac{1}{A \eta_{A}^{2}}-\frac{1}{B \eta_{B}^{2}}\right]^{(-1)} \quad \text { and } & \bar{R}=
\end{array}
$$$$
(R * \bar{S}) / S \text {. }
$$

Using Eqs. (29) and (30), the scattering amplitudes of quark and nucleon pictures can be obtained by performing the integration in Eqs. (2) and (3) numerically. If we take cluster configuration $M_{A}=A, M_{B}=B$ and $M_{N}=1$, the integration gives the analytical result of the nucleon model given by:

$$
F_{A B}(q)=1+c_{A} c_{B}\left\{\sum_{V_{1}=1}^{M_{1}} \sum_{\lambda_{V_{1}}}(-g)^{V_{1}} * T_{1}\left(V_{1}, \lambda_{V_{1}}\right) *\right.
$$

$$
\left.\frac{\bar{R}\left(V_{1}, \lambda_{V_{1}}, \Delta_{i \alpha, j \delta}\left(V_{1}, \lambda_{V_{1}}\right)\right)}{2 \bar{S}\left(V_{1}, \lambda_{V_{1}}, \Delta_{i \alpha, j \delta}\left(V_{1}, \lambda_{V_{1}}\right)\right)} \exp \left[-\frac{q^{2}}{4 \bar{S}\left(V_{1}, \lambda_{V_{1}}, \Delta_{i \alpha, j \delta}\left(V_{1}, \lambda_{V_{1}}\right)\right)}\right]\right\}
$$

where $\quad V_{1}=\sum_{i=1}^{M_{A}} \sum_{j=1}^{M_{B}} \Delta_{i j}\left(V_{1}, \lambda_{V 1}\right)$

The angular distribution of the total elastic scattering amplitude of Eq.(1) in hybrid model is then given by:

$$
\frac{d \sigma(\vec{q})}{d \Omega}=\left|F_{A B}(\vec{q})\right|^{2}
$$

\section{RESULTS AND DISCUSSION}

In section (2), the composite-composite elastic collisions have been analyzed in a hybrid model combining two pictures, namely the quark and nucleonic models of their structures and considering the full Glauber series of the multiple scattering between their constituents as nucleons and as quarks. Analytic formulas for the nuclear phase shift function of these pictures are developed using single Gaussian parameterizations for the quark/nucleon densities and elemental scattering amplitudes. The hybrid model developed here is applied to calculate the differential cross sections of $\alpha p$ elastic scattering at $\sqrt{s}=89 \mathrm{GeV}$ and $\alpha \alpha$ elastic scattering at $\sqrt{s}=126 \mathrm{GeV}$. For comparison, the corresponding results of the quark model and the conventional nucleon model are also evaluated independently. The ingredients needed to perform these calculations are the parameters associated with the $q q$ and the $N N$ scattering amplitudes $\left(\sigma, \varepsilon\right.$ and $\left.\beta^{2}\right)$ and the quark/nucleon densities $\left(\eta_{A}, \eta_{B}\right)$. The $q q$ parameters used are obtained by fitting the $N N$ data of corresponding energies and the values of both are listed in Table (1). 
Table (1): The parameters of the $q q$ and $N N$ scattering amplitudes

\begin{tabular}{ccccccccc}
\hline \multirow{2}{*}{$\sqrt{s}$} & \multicolumn{2}{c}{$\sigma\left(\mathrm{fm}^{2}\right)$} & \multicolumn{2}{c}{$\varepsilon$} & \multicolumn{2}{c}{$\beta^{2}\left(\mathrm{fm}^{2}\right)$} & \multicolumn{2}{c}{ Ref. No. } \\
\cline { 2 - 9 }$(\mathrm{GeV})$ & $\sigma_{q q}$ & $\sigma_{\mathrm{NN}}$ & $\varepsilon_{\mathrm{qq}}$ & $\varepsilon_{\mathrm{NN}}$ & $\beta^{2}{ }_{\mathrm{qq}}$ & $\beta^{2}{ }_{\mathrm{NN}}$ & $q q$ & $N N$ \\
\hline 30.7 & 0.5352 & 4.014 & 0.045 & 0.037 & 0.083 & 0.47505 & {$[55]$} & {$[57]$} \\
44.7 & 0.5555 & 4.179 & 0.075 & 0.062 & 0.105 & 0.4984 & {$[56]$} & {$[58]$} \\
\hline
\end{tabular}

The values of the parameters $\eta_{A}, \eta_{B}$, after correcting for the effects of the finite proton-size and the c. m. recoil, are given by:

$$
\eta_{\gamma}^{2}=\frac{3}{2}\left(\frac{(1-1 / \gamma)}{\left\langle r_{\gamma}^{2}\right\rangle-\left\langle r_{p}{ }^{2}\right\rangle}\right) \quad, \quad \gamma=A, B,
$$

Where $\left\langle r_{\gamma}{ }^{2}\right\rangle$ and $\left\langle r_{p}{ }^{2}\right\rangle$ are the mean square radii of the nucleus and the proton, respectively and their values were reported in an earlier publication[55].

The numbers $\left(\mathrm{M}_{\mathrm{A}}, \mathrm{M}_{\mathrm{B}}, \mathrm{M}_{\mathrm{N}}\right)$, determining the configuration of the cluster structure assumed in each system, are taken as follows: For $\alpha-p$ case, the cluster configuration of the quark model is taken to be $M_{A}=4$, $M_{B}=1$ and $M_{N}=3$ while the configuration for the nucleon model gives $\mathrm{M}_{\mathrm{A}}=4, \mathrm{M}_{\mathrm{B}}=1$ and $\mathrm{M}_{\mathrm{N}}=1$. For $\alpha-\alpha$ case, the cluster configuration of the quark model is taken to be $\mathrm{M}_{\mathrm{A}}=4, \mathrm{M}_{\mathrm{B}}=4$ and $\mathrm{M}_{\mathrm{N}}=3$ while the configuration for the nucleon model gives $\mathrm{M}_{\mathrm{A}}=4, \quad \mathrm{M}_{\mathrm{B}}=4$ and $\mathrm{M}_{\mathrm{N}}=1$. The orbits, lengths and $\Delta$-matrices of the groups $G_{1}=$ $S_{M_{A}} \otimes S_{M_{B}}$ and $\quad G_{2}=S_{M_{N}} \otimes S_{M_{N}} \quad$ represent these structures are given in appendix (I) .

The application of these ingredients started by calculating the differential cross-section of the $\quad \alpha-$ $p$ elastic scattering at $\sqrt{S}=89 \mathrm{GeV}$. The results are shown in Fig.(1). In this Figure, the full Glauber series calculation obtained from the hybrid model (solid curve), the quark model (dashed curve) and the nucleon model (dotted curve) in comparison with the experimental data were presented. The comparison shows that the calculations obtained from the three models are identical in reproducing the scattering data over the range of momentum transfer $0.0<-t<0.3(\mathrm{GeV} / \mathrm{c})^{2}$. The excellent agreement obtained over the smaller values of momentum transfer is expected because the Glauber theory is a good approximation at forward angles. For $-t \geq 0.3(\mathrm{GeV} / \mathrm{c})^{2}$, the results obtained from the three models are significantly different in their agreements. The results of the hybrid model (solid curve) are greatly successful in describing the scattering data

up to $-t \approx 0.73(\mathrm{GeV} / \mathrm{c})^{2}$ while the predictions of the other models are failing in reproducing the data for $-t \geq$ $0.3(\mathrm{GeV} / \mathrm{c})^{2}$. Either the predictions of the quark model (dashed curve) or the results of the nucleon model (dotted curve) underestimates the existing data beyond $-t=$ $0.3(\mathrm{GeV} / \mathrm{C})^{2}$. Obviously, we can notice the advantage of the nucleon model calculations over the quark model ones in explaining the experimental data, but this advantage is expected because the full Glauber series of the nucleon-nucleon scatterings is calculated more rigorously than quark-quark interactions one. However, our calculation of $\alpha-p$ scattering using the hybrid model developed here with full Glauber series of quark-quark and nucleon-nucleon multiple scatterings gives slightly better agreement at large momentum transfer than that obtained in a previous investigation[56] using different analysis for the hybrid model.

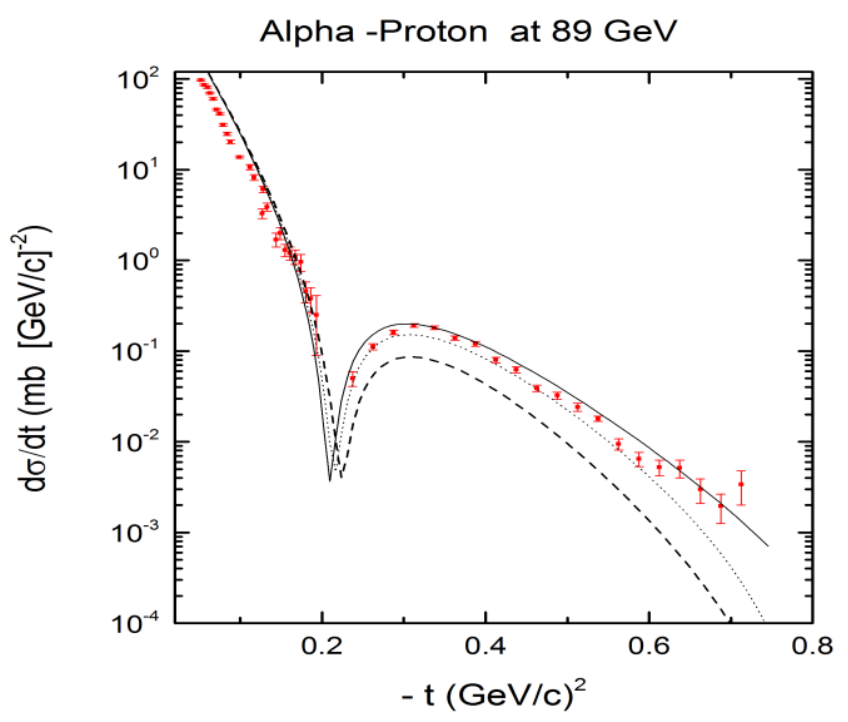

Fig. (1): Differential cross-section of $\alpha-p$ elastic collision at $\sqrt{s}=89 \mathrm{GeV}$. Full Glauber series evaluation results of hybrid, quark and nucleon models are compared with the experimental data. The dashed curve is the quark model results. The dotted curve is the nucleon model results. The solid curve is the hybrid model results. The experimental data are from Ref. [12]. 
The results of $\alpha-\alpha$ elastic scattering at $\sqrt{s}=126 \mathrm{GeV}$ is presented in Fig.(2), where the solid curve represents the hybrid model calculations, the dashed curve displays the pure quark model predictions and the dotted curve stands for the results of the nucleon model. The curves in Fig.(2) are labeled as in Fig. (1). The results in Fig. (2) show that the hybrid model (solid curve) improves in general the theoretical descriptions given before by the quark model (dashed curve) and nucleon model (dotted curve) calculations, but with inferior quality than the fitting obtained for $\alpha$-p elastic scattering case. The results of the three approaches are also almost identical and reasonable, fairly agree with the scattering data over the range of momentum transfer $0.05<-t<$ $0.25(\mathrm{GeV} / \mathrm{C})^{2}$, and beyond this range, their predictions are clearly of less well founded agreements. For momentum transfers $-t \geq 0.25(\mathrm{GeV} / \mathrm{c})^{2}$, the results of hybrid model have shown closer values to the experimental data than those obtained from the nucleon and the quark models. Although the discrepancies between the sets of measurements at forward and large angles make the comparisons difficult, the full Glauber series calculation using the hybrid model developed in the present study gives a better agreement for $\alpha-\alpha$ scattering than that obtained earlier [57] using different analysis for the hybrid model. Finally, it could be ensured that the quark degrees of freedom in the interior quark region really play an important role in improving the theoretical descriptions of $\alpha-p$ and $\alpha-$ $\alpha$ experimental data, especially at large momentum transfers. Alpha - Alpha at $126 \mathrm{GeV}$

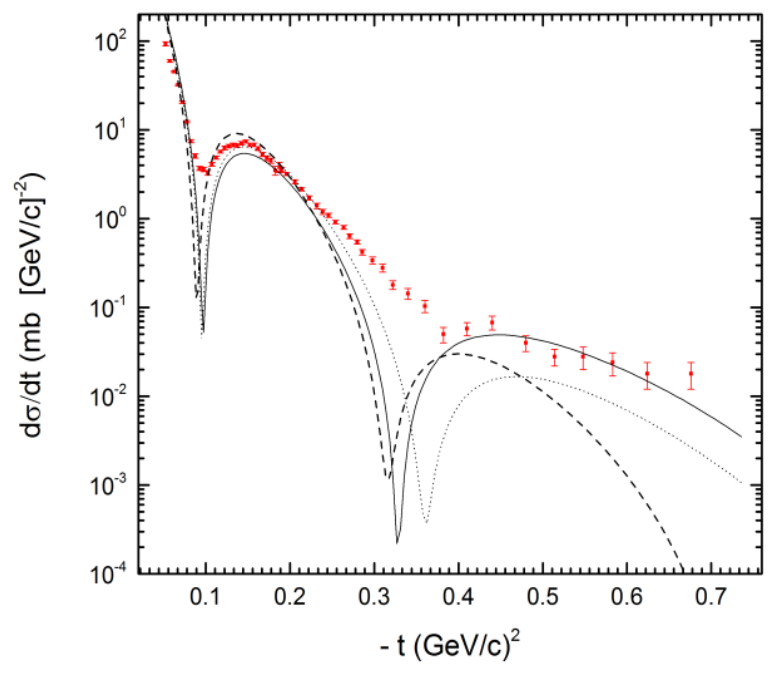

Fig. (2): Differential cross-section of $\alpha-\alpha$ elastic collision at $\sqrt{s}=126 \mathrm{GeV}$. Full Glauber series calculation results of hybrid, quark and nucleon models are compared with the experimental data. The dashed curve is the quark model results. The dotted curve is the nucleon model results. The solid curve is the hybrid model results. The experimental data are from Refs. [14,15].

\section{CONCLUSION}

The elastic collisions between two composite systems at very large energies have been investigated by developing a hybrid model combines the quark and nucleon pictures including the full Glauber multiple scattering series of their constituents. Using Gaussian forms for the quark/nucleon densities and scattering amplitudes, analytic formulas have been developed to calculate $\alpha-p$ and $\alpha-\alpha$ interactions at center of mass energies 89 and $126 \mathrm{GeV}$, respectively. The positive findings and negative criticisms of the present study could be summarized in a united conclusion as follows:

The hybrid model combining the full Glauber series of the quark and nucleon models improves the prediction of the Glauber theory than applying this theory by using the quark and nucleon models independently. The hybrid model is successful in reproducing the experimental data of high energy $\alpha-p$ and $\alpha-\alpha$ elastic scattering, at large momentum transfers, where the high orders of multiple scattering are dominant. We can conclude that the quark degrees of freedom in the interior quark region really play an important role in improving the theoretical description of experimental data. In particular, the inclusion of the full series enhances the applicability of the hybrid model at a large momentum transfer over both quark and nucleon structures.

The common criticism against present calculations and those previously employed in previous studies [55-58] is that the quark model always underestimates the predicted cross sections more than the nucleon model at large momentum transfers. Surely, considering the structure of the composite systems as quarks adds a larger number of scattering centers rather than their number as nucleons and consequently enlarges the number of the higher order multiple scattering terms which have an effective role at a large momentum transfer. Thus, the underestimation means that treating the composite scattering as pure quark-quark interactions has added unrealistic higher order scattering terms interfere more destructively than those of nucleon-nucleon collisions. This justification has been tested before using the approach developed by M. W. Hsing et al. [56] and confirmed by the present one.

Our systematic future improvements to complete this study in the same direction are suggested as follows:

First, the full Glauber multiple scattering series of quark model can be calculated with a more elaborate technique as the one proposed by El-Gogary [59]. Second, as an alternative, a more realistic form for the quark/ nucleon scattering amplitudes can be used such as sum Gaussian or harmonic oscillator shapes, rather than the Gaussian form. Both recommended improvements 
may lead to a better description and may assess the question about the predictive power of the proposed hybrid model seriously.

\section{Appendix I}

This appendix contains the Tables in which the orbits, lengths and $\Delta$ - matrices employed in the present calculations are presented. They were obtained by enumerating and investigating all the possible combinations of collisions according to their permutation [44]. In the preset work, the elastic collisions $\alpha-p$ and $\alpha$ $\alpha$ have been studied under their quark and nucleon structures. The orbits, lengths and $\Delta$-matrices of the groups $G_{1}=S_{M_{A}} \otimes S_{M_{B}}$ and $G_{2}=S_{M_{N}} \otimes S_{M_{N}}$ (defined in section 2) representing the multiple scattering of these reactions depend on the assumed cluster configuration. For the $\alpha$-p scattering, the quark model yields cluster configuration $M_{A}=4, M_{B}=1$ and $M_{N}=3$, while the nucleon model satisfies the cluster configuration $\mathrm{M}_{\mathrm{A}}=4, \mathrm{M}_{\mathrm{B}}=1$ and $M_{N}=1$. For $\alpha-\alpha$ scattering, the quark model yields the cluster configuration $\mathrm{M}_{\mathrm{A}}=\mathrm{M}_{\mathrm{B}}=4$ and $\mathrm{M}_{\mathrm{N}}=3$. However, the nucleon model gives the cluster configuration $M_{A}=M_{B}=1$ and $M_{N}=4$. For the sake of brevity, only the Tables of the non-similar groups were given.

Table (1): Orbits, lengths and $\Delta$ - matrices for $\mathrm{G}=S_{4} \otimes S_{1}$

\begin{tabular}{|c|c|c|c|}
\hline $\mathbf{V}$ & $\lambda_{\mathbf{v}}$ & $\mathbf{T}\left(\mathbf{V}, \boldsymbol{\lambda}_{\mathbf{v}}\right)$ & $\boldsymbol{\Delta}\left(\mathbf{V}, \boldsymbol{\lambda}_{\mathbf{v}}\right)$ \\
\hline 1 & 1 & 4 & 1000 \\
2 & 1 & 6 & 1100 \\
\hline
\end{tabular}

Total number of orbits (including the orbits not shown) $=4$

Table (2): Orbits, lengths and $\Delta$ - matrices for $S_{3} \otimes S_{3}$

\begin{tabular}{|c|c|c|c|}
\hline $\mathbf{V}$ & $\boldsymbol{\lambda}_{\mathbf{v}}$ & $\mathbf{T}\left(\mathbf{V}, \boldsymbol{\lambda}_{\mathbf{v}}\right)$ & $\boldsymbol{\Delta}\left(\mathbf{V}, \boldsymbol{\lambda}_{\mathbf{v}}\right)$ \\
\hline 1 & 1 & 9 & 10000000 \\
2 & 1 & 18 & 110000000 \\
2 & 2 & 18 & 100010000 \\
3 & 1 & 6 & 111000000 \\
3 & 2 & 36 & 110001000 \\
3 & 3 & 36 & 110100000 \\
3 & 4 & 6 & 100010001 \\
4 & 1 & 36 & 111100000 \\
4 & 2 & 36 & 110101000 \\
4 & 3 & 9 & 110110000 \\
4 & 4 & 9 & 110100001 \\
4 & 5 & 011100100 \\
\hline
\end{tabular}

Total number of orbits (including the orbits not shown) $=25$
Table (3): Orbits, lengths and $\Delta$ - matrices for $S_{4} \otimes S_{4}$

\begin{tabular}{|c|c|c|c|}
\hline V & $\lambda_{\mathrm{v}}$ & $\mathbf{T}\left(\mathbf{V}, \lambda_{\mathbf{V}}\right)$ & $\Delta\left(\mathbf{V}, \lambda_{\mathrm{V}}\right)$ \\
\hline 1 & 1 & 16 & 1000000000000 \\
\hline 2 & 1 & 48 & 1100000000000 \\
\hline 2 & 2 & 72 & 100001000000000 \\
\hline 3 & 1 & 32 & 11100000000000 \\
\hline 3 & 2 & 288 & 110000100000000 \\
\hline 3 & 3 & 144 & 11001000000000 \\
\hline 3 & 4 & 96 & 1000010000100000 \\
\hline 4 & 1 & 8 & 11110000000000 \\
\hline 4 & 2 & 288 & 11101000000000 \\
\hline 4 & 3 & 96 & 111000010000000 \\
\hline 4 & 4 & 288 & 110010100000000 \\
\hline 4 & 5 & 72 & 110000110000000 \\
\hline 4 & 6 & 288 & 11000010001000 \\
\hline 4 & 7 & 36 & 11001100000000 \\
\hline 4 & 8 & 576 & 110010000100000 \\
\hline 4 & 9 & 144 & 011010001000000 \\
\hline 4 & 10 & 24 & 1000010000100001 \\
\hline 5 & 1 & 96 & 11111000000000 \\
\hline 5 & 2 & 288 & 111011000000000 \\
\hline 5 & 3 & 288 & 111010010000000 \\
\hline 5 & 4 & 576 & 111010001000000 \\
\hline 5 & 5 & 576 & 111010000010000 \\
\hline 5 & 6 & 96 & 011110001000000 \\
\hline 5 & 7 & 576 & 1100101000010000 \\
\hline 5 & 8 & 576 & 110000111000000 \\
\hline 5 & 9 & 144 & 111010001000000 \\
\hline 5 & 10 & 144 & 110011000010000 \\
\hline 5 & 11 & 576 & 011011001000000 \\
\hline 5 & 12 & 288 & 1100100000100001 \\
\hline 5 & 13 & 144 & 01101001000001 \\
\hline 6 & 1 & 144 & 111111000000000 \\
\hline
\end{tabular}


Table (3): (Continued)

\begin{tabular}{|c|c|c|c|}
\hline $\mathbf{V}$ & $\lambda v$ & $\mathbf{T}\left(\mathbf{V}, \lambda_{\mathrm{v}}\right)$ & $\Delta(\mathbf{V}, \lambda \mathbf{v})$ \\
\hline 6 & 2 & 96 & 111110001000000 \\
\hline 6 & 3 & 288 & 11111000100000 \\
\hline 6 & 4 & 48 & 111011100000000 \\
\hline 6 & 5 & 144 & 111011010000000 \\
\hline 6 & 6 & 576 & 111010011000000 \\
\hline 6 & 7 & 576 & 111011000100000 \\
\hline 6 & 8 & 576 & 111011000010000 \\
\hline 6 & 9 & 1152 & 111001011000000 \\
\hline 6 & 10 & 575 & 011111001000000 \\
\hline 6 & 11 & 192 & 111010001000010 \\
\hline 6 & 12 & 288 & 011110001001000 \\
\hline 6 & 13 & 576 & 1100101001010000 \\
\hline 6 & 14 & 144 & 1100110000110000 \\
\hline 6 & 15 & 288 & 0011110010000100 \\
\hline 6 & 16 & 576 & 111010000100001 \\
\hline 6 & 17 & 576 & 111011001000000 \\
\hline 6 & 18 & 144 & 111010001000001 \\
\hline 6 & 19 & 16 & 0111100010001000 \\
\hline 6 & 20 & 96 & 1100101001100000 \\
\hline 6 & 21 & 72 & 1100110000100001 \\
\hline 6 & 22 & 576 & 1100101001000001 \\
\hline 6 & 23 & 288 & 0101101001001000 \\
\hline 7 & 1 & 96 & 111111100000000 \\
\hline 7 & 2 & 576 & 111111001000000 \\
\hline 7 & 3 & 576 & 111111000100000 \\
\hline 7 & 4 & 288 & 1111100010000100 \\
\hline 7 & 5 & 192 & 1111100001000010 \\
\hline 7 & 6 & 288 & 111011101000000 \\
\hline 7 & 7 & 576 & 111011011000000 \\
\hline 7 & 8 & 96 & 1110111000010000 \\
\hline 7 & 9 & 1152 & 1110110010010000 \\
\hline
\end{tabular}

Table (3): (Continued)

\begin{tabular}{|c|c|c|c|}
\hline $\mathbf{V}$ & $\lambda_{\mathrm{V}}$ & $\mathbf{T}\left(\mathbf{V}, \lambda_{\mathrm{V}}\right)$ & $\Delta\left(\mathbf{V}, \lambda_{\mathrm{v}}\right)$ \\
\hline 7 & 10 & 576 & 111011000110000 \\
\hline 7 & 11 & 576 & 1110110000100001 \\
\hline 7 & 12 & 576 & 1110110100100000 \\
\hline 7 & 13 & 288 & 101111001100000 \\
\hline 7 & 14 & 576 & 101111001000100 \\
\hline 7 & 15 & 576 & 110110100110000 \\
\hline 7 & 16 & 288 & 110111000100010 \\
\hline 7 & 17 & 576 & 1110100100100100 \\
\hline 7 & 18 & 1152 & 011111000101000 \\
\hline 7 & 19 & 16 & 1111100010001000 \\
\hline 7 & 20 & 288 & 111011001010000 \\
\hline 7 & 21 & 1152 & 111011001000001 \\
\hline 7 & 22 & 144 & 0111110010001000 \\
\hline 7 & 23 & 576 & 111010011000100 \\
\hline 7 & 24 & 144 & 1100110000110010 \\
\hline 7 & 25 & 576 & 0110110010010010 \\
\hline 7 & 26 & 96 & 0110110010100001 \\
\hline 8 & 1 & 12 & 111111110000000 \\
\hline 8 & 2 & 576 & 111111101000000 \\
\hline 8 & 3 & 192 & 111111100010000 \\
\hline 8 & 4 & 144 & 111111001100000 \\
\hline 8 & 5 & 576 & 111111001010000 \\
\hline 8 & 6 & 144 & 1111110000110000 \\
\hline 8 & 7 & 288 & 111111001000100 \\
\hline 8 & 8 & 1152 & 111111001000010 \\
\hline 8 & 9 & 288 & 1111110000100001 \\
\hline 8 & 10 & 288 & 111011011100000 \\
\hline 8 & 11 & 288 & 1110110100110000 \\
\hline 8 & 12 & 288 & 111011101000001 \\
\hline 8 & 13 & 48 & 1110111000010001 \\
\hline
\end{tabular}


Table (3):(Continued)

\begin{tabular}{|c|c|c|c|}
\hline $\mathbf{V}$ & $\lambda_{\mathrm{v}}$ & $\mathbf{T}\left(\mathbf{V}, \lambda_{\mathrm{v}}\right)$ & $\Delta\left(\mathbf{V}, \lambda_{\mathbf{v}}\right)$ \\
\hline 8 & 14 & 288 & 1110111010010000 \\
\hline 8 & 15 & 576 & 1110110000110001 \\
\hline 8 & 16 & 1152 & 1011110010100100 \\
\hline 8 & 17 & 576 & 1110100100110100 \\
\hline 8 & 18 & 288 & 1111011010001000 \\
\hline 8 & 19 & 1152 & 1101111010100000 \\
\hline 8 & 20 & 288 & 0111111010001000 \\
\hline 8 & 21 & 1152 & 1101111010000010 \\
\hline 8 & 22 & 288 & 1110110100100001 \\
\hline 8 & 23 & 576 & 1011110011000010 \\
\hline 8 & 24 & 144 & 1111110010001000 \\
\hline 8 & 25 & 144 & 1110111011000000 \\
\hline 8 & 26 & 144 & 1110110110000100 \\
\hline 8 & 27 & 288 & 1110110010100001 \\
\hline 8 & 28 & 576 & 1011011011001000 \\
\hline 8 & 29 & 576 & 1110110010010010 \\
\hline 8 & 30 & 288 & 0111101011001000 \\
\hline 8 & 31 & 18 & 1100110000110011 \\
\hline 8 & 32 & 72 & 0110110010010011 \\
\hline
\end{tabular}

Total number of orbits (including the orbits not shown) $=191$

In these Tables, the first column represents the order of multiple scattering $\mathrm{V}$ which ranges from 1 to $\mathrm{m} \times \mathrm{n}$ , while $\lambda_{\mathrm{V}}$ in the second column represents the serial index used to number the orbits of order V. The third column represents the length of the orbit $\mathrm{T}\left(\mathrm{V}, \lambda_{\mathrm{V}}\right)$. In the fourth column the $\mathrm{m} \times \mathrm{n}$ - digit binary numbers give the $\Delta$ - matrices of the group $G=S_{m} \otimes S_{n}$. The first n-digits are the elements $\Delta_{1 \mathrm{i}}, \mathrm{i}=1,2, . ., \mathrm{n}$; the next $\mathrm{n}$ digits are $\Delta_{2 \mathrm{i}}, \ldots$, and the last $\mathrm{n}$-digits are $\Delta_{\mathrm{mi}}$. By symmetry, the orbits, lengths and $\Delta$ - matrices for V's which are not shown in the Tables could be easily deduced from the Tables. This is carried out using the results for order $\mathrm{V}^{\prime}=\mathrm{m} \times \mathrm{n}-\mathrm{V}$ and interchange the 0 's and 1's of $\Delta\left(\mathrm{V}^{\prime}, \lambda_{\mathrm{V}}\right)$. The indices $\lambda_{\mathrm{V}}$ and $\lambda_{\mathrm{V}}$ are the same and the lengths $T\left(\mathrm{~V}^{\prime}, \lambda_{\mathrm{V}^{\prime}}\right)$ and $\mathrm{T}\left(\mathrm{V}^{\prime}, \lambda_{\mathrm{V}^{\prime}}\right)$ are equal. The matrix $\Delta(m \times n, 1)$ has elements $\Delta_{\mathrm{ij}}$ equal 1 .

\section{REFERENCES}

[1] Faldt, G., Pilkuhn, H., Sclaile, H.G. (1974), Nucleusnucleus collisions at relativistic energies, Ann. Phys., 82(2), 326.

[2] Andreev, I.V., Chernov, A.V. (1978), Interaction of heavy relativistic nuclei, Sov. J. Nucl. Phys. (Engl. Transl. Yadernaya Fizika), 28(2), 477.

[3] Andreev, I. V., Khein, L. A. (1978), Calculation of the phase shifts and scattering cross sections of heavy relativistic nuclei, Sov. J. Nucl. Phys. (Engl. Transl.), 28(6), 770 .

[4] Gasparyan, A., Cheplakov, A., Shabel'skii, Y. (1981), Distributions in the number of interacting nucleons in collisions of relativistic nuclei, Sov. J. Nucl. Phys.; ISS, v. 34(5), 739.

[5] Alkhazov, G.D., Bauer, T., Bertini, R., et al. (1977), Elastic and inelastic scattering of $1.37 \mathrm{GeV} \alpha$-particles from $40,42,44,48 \mathrm{Ca}$, Nucl Phys., A280, 365.

[6] Lenzi, S. M., Vitturi, A., Zardi, F. (1989), Systematic analysis of heavy-ion reaction data in terms of an eikonal approach: Elastic and inelastic scattering, Phys. Rev. C 40, 2114.

[7] Chauvin, J., Lebrun, D., Lounis, A., Buenerd, M. (1983), Low and intermediate energy nucleus-nucleus elastic scattering and the optical limit of Glauber theory, Phys. Rev., C28, 1970.

[8] Morsch, H.P., Boivin, M., Boyard, J.L., et al. (1994), Study of elastic $\alpha$-scattering from $2 \mathrm{H}$ and ${ }^{12} \mathrm{C}$ at $\mathrm{E}_{\alpha}=4.2 \mathrm{GeV}, \mathrm{Z}$. Physik A-Hadrons and Nuclei, 350, 167.

[9] Bartnitzky, G., et al. (1996), Model-unrestricted nucleusnucleus scattering potentials from measurement and analysis of ${ }^{16} 0+{ }^{16} 0$ scattering, Phys. Lett., B365, 23.

[10] Ji-feng Liu, Yu-shun Zhang, Chao-yun Yang, Jun-feng Shen, Robson, B. A. (1996), Elastic and inelastic scattering of $1.37 \mathrm{GeV} \alpha$ particles from

${ }^{12} \mathrm{C}$ and ${ }^{40,42,44,48} \mathrm{Ca}$, Phys. Rev., C 54, 2509.

[11] Lombard, R. J., Maillet, J. P. (1990), Phase variation of the nucleon-nucleon amplitude and the spin observables in proton-4He elastic scattering, Phys. Rev., C41, R1348.

[12] Schubert, K. R. (1980), Tables on Nucleon-Nucleon Scattering, Vol.9, Springer, New York, p. 273.

[13] Amaldi, U., Schubert, K. R. (1980), Impact Parameter Interpretation of Proton Proton Scattering 
from a Critical Review of All ISR Data, Nucl.Phys., B166, 301-320

[14] CERN-Napoli-Pisa-Stony Brook Collaboration, Ambrosio, M., et al. (1982), Measurements of elastic scattering in alpha-alpha and alpha-proton collisions at the CERN intersecting storage rings, Phys.Lett., $113 \mathrm{~B}, 347$.

[15] Bell, W., Braune, K., Claesson, G., et al. (1982), Measurement of $\alpha \alpha$ and $\alpha p$ Elastic Scattering at the cern ISR, Phys. Lett., 117 B, 131.

[16] Satta, L., Duflo, J., Plouin, F., Picozza, P., Goldzahl, L., Banaigs, J., Frascaria, H., Fabbri, F.L., Codino, A., Berger, J., Boivin, M., Berthet, P. (1984), Errata: Elastic scattering of $\alpha$ particles on light nuclei at $\mathrm{P}_{\alpha}=7 \mathrm{GeV} / \mathrm{c}$, Phys. Lett., B139, 263.

[17] Amos, N., et al. (1985), Measurement of small-angle antiproton-proton and proton-proton elastic scattering at the CERN intersecting storage rings, Nucl. Phys., B262, 689.

[18] Desgrolard, P., Giffon, M., Martynov, E. (2000), Elastic $p p$ and $p \bar{p}$ scattering in the modified additive quark model, Eur. Phys. J. C 18, 359-367.

[19] TOTEM Collaboration, Antchev, G., et. al. (2011), Proton-proton elastic scattering at the LHC energy of $\sqrt{s}=7 \mathrm{TeV}$, Europhys. Lett. 95, 41001 .

[20] TOTEM Collaboration, Antchev, G., et. al. (2011), First measurement of the total proton-proton cross-section at the LHC energy of $\sqrt{s}=7 \mathrm{TeV}$, Europhys. Lett. $96,21002$.

[21] TOTEM Collaboration, Antchev, G., et. al. (2012), Measurement of the forward charged-particle pseudorapidity density in $p p$ collisions at $\sqrt{s}=$ $7 \mathrm{TeV}$ with the TOTEM experiment, Europhys. Lett. 98,31002 .

[22] TOTEM Collaboration, Antchev, G., et. al. (2013), Measurement of proton-proton elastic scattering and total cross-section at at $\sqrt{s}=7 \mathrm{TeV}$, Europhys. Lett. 101, 21002.

[23] TOTEM Collaboration, Antchev, G., et. al. (2013), Measurement of proton-proton inelastic scattering cross-section at $\sqrt{ } s=7 \mathrm{TeV}$, Europhys. Lett. 101, 21003.

[24] TOTEM Collaboration, Antchev, G., et. al. (2013), Luminosity-independent measurements of total, elastic and inelastic cross-sections at $\sqrt{s}=7 \mathrm{TeV}$, Europhys. Lett. 101, 21004.
[25] TOTEM Collaboration, Antchev, G., et. al. (2015), Evidence for non-exponential elastic proton-proton differential cross-section at low $|t|$ and $\sqrt{ } s=8$ TeV by TOTEM, Nucl. Phys., B 899, 527.

[26] Khoze, V. A., Martin, A. D., Ryskin, M. G. (2015), $t$ dependence of the slope of the high energy elastic $p p$ cross section, J. Phys. G: Nucl. Part. Phys., 42, 025003.

[27] TOTEM Collaboration, Antchev, G., et. al. (2016), Measurement of elastic $p p$ scattering at $\sqrt{s}=$ $8 \mathrm{TeV}$ in the Coulomb-nuclear interference region: determination of the $\rho$-parameter and the total crosssection, Eur. Phys. J., C 76, 661.

[28] TOTEM Collaboration, Antchev, G., et. al. (January 25, 2018), First measurement of elastic, inelastic and total cross-section at $\sqrt{s}=13 \mathrm{TeV}$ by TOTEM and overview of cross-section data at LHC energies, CERN-PH-EP-2017-231.

[29] Glauber, R. J. (1959), Lectures on Theoretical Physics, (Editors: Brittin, W. E., and Dunham, L. C.), Interscience, 1, New York, p. 315.

[30] Franco, V., Glauber, R. J. (1966), High-Energy Deuteron Cross Sections, Phys. Rev., 142, 1195. [31] Glauber, R. J.,Franco, V. (1967), High-Energy Deuteron Cross Sections: Charge-Exchange Effects, Phys. Rev., 156, 1685.

[32] Toshimi Adachi, Tsuneyuki Kotani (1965), An Impact Parameter Formalism, Prog. Theor. Phys. Suppl., E65, 316.

[33] Toshimi Adachi, Tsuneyuki Kotani (1966), Unitarity Relation in an Impact Parameter Representation, Phys. Suppl. 37-38, 297.

[34] Toshimi Adachi (1966), An Impact Parameter Formalism. II: High Energy Elastic Scattering, Theor. Phys., 35, 463.

[35] Toshimi Adachi, Tsuneyuki Kotani (1966), An Impact Parameter Formalism. III: Strong Absorption Model, Theor. Phys., 35, 485.

[36] Toshimi Adachi, Tsuneyuki Kotani (1968), An Impact Parameter Representation of the Scattering Problem, Prog. Theor. Phys., 39, 430.

[37] Toshimi Adachi, Tsuneyuki Kotani (1968), An Impact Parameter Representation of the Scattering Problem, Prog. Theor. Phys., 39, 785.

[38] Islam , M. M. (1968), Lectures in Theoretical Physics, Vol. 10B, (edited by A. O. Barut and W. E. Brittin), Gordon and Breach, pp. 97. 
[39] Islam, M. M. (1976), Impact parameter representation from the Watson-Sommerfeld transform, Nucl. Phys., B104, 511.

[40] Varma, G.K. (1987), Scattering of $\alpha$-particles at medium energies, Nuclear Physics, A294, 465.

[41] Victor Franco and Amouzou Tekou (1977), Highenergy heavy-ion scattering and the optical phase shift function, Phys. Rev., C16, 658.

[42] Victor Franco and Girish K. Varma (1978), Collisions between composite particles at medium and high energies, Phys. Rev., C18, 349.

[43] Viollier,R. D.,Turtschi, E. (1980), Elastic alphanucleus scattering at high energies, Ann. Phys.(N. Y.), Vol 124, Issue 2, 290.

[44] Yi-Chun Yin, Zhen-Qiang Tan, Ke-Zhong Chen (1985), METHOD FOR CALCULATING THE COMPLETE EXPANSION OF THE GLAUBER AMPLITUDE FOR NUCLEUS NUCLEUS SCATTERING, Nucl. Phys. A440, 685.

[45] YIN Yi-chun, TAN Zhen-qiang, CHEN Ke-zhong (1986), High energy nucleus-nucleus elastic scattering, Chinese Phys., 6, 93; Yi-Chun Yin, Zhen-Qiang Tan, Ke-Zhong Chen (1985), HIGHENERGY NUCLEUS NUCLEUS ELASTIC SCATTERING, Phys. Energ. Fort. Phys. Nucl., 9, 569. [46] Usmani, A. A., Ahmad, I., Usmani, Q. N. (1989), $\alpha{ }^{4} \mathrm{He}$ elastic scattering at high energies, Phys. Rev., C39, 1182.

[47] Victor Franco, Yichun Yin (1989), Elastic collisions between light nuclei and the phase variation of the nucleon-nucleon scattering amplitude, Phys. Rev., C 34, 608.

[48] Tan Zhen-Qiang, Lin Zheng-Jin, Yan Pan-Ning, Wu Chong-En (1990), An Integral Formula for calculating the Glauber multiple scattering amplitude of composite particles, J. Phys. G: Nucl. Part. Phys., 16, 1697.

[49] Zhong-Jin Lin, Shu-Xia Yu, Xiang-Zhong Huang, Chong-En Wu (1991), Elastic scattering of composite particles with multiconstituents, J. Phys. G: Nucl. Part. Phys., 17, 1159.

[50] Franco, V., Tekou, A. (1988), Optical model for medium and high energy hadron-nucleus collisions, Phys. Rev., C37, 1097; Lombard, R. J. , Maillet, J. P., Phase variation of the nucleon-nucleon amplitude and the spin observables in proton-4He elastic scattering, Phys. Rev. C 41, R1348.

[51] Huang Xiang Zhong, (1995), Method for calculating elastic scattering between two composite many-body systems at high energies, Phys. Rev., C51, 2700.

[52] Shalaby, A. S., El-Gogary, M. M. H., Hassan, M. Y. M. (1997), Comment on "Method for calculating elastic scattering between two composite many-body systems at high energies”, Phys. Rev., C 56, 2889.

[53] El-Gogary, M. M. H., Shalaby, A. S., Hassan, M. Y. M. (1998), Elastic scattering between two cluster nuclei $(A, \mathrm{~B}>\sim 4)$ at medium and high energies, Phys. Rev., C 58, 3513.

[54] El-Gogary, M.M.H., Shalaby, A.S., Hassan, M.Y.M., Hegazy, A.M. (2000), Full Glauber series analysis for elastic scattering with consistent centerof-mass correlations, Phys. Rev., C 61, 044604.

[55] Franco, V. (1987), QUARK MODEL FOR NUCLEUS NUCLEUS COLLISIONS, Phys. Rev., C35, 1328.

[56] Ma, Wei-Hsing, Huang, Chao-Hui,Wang, DeAn,(1989), COMPOSITE PARTICLE SCATTERING IN A HYBRID QUARK HADRON MODEL, Nucl. Phys., A496, 729.

[57] Huang Chao-hui, Wang De-an, Ma Wei-hsing. (1990), Elastic $4 \mathrm{He}+4 \mathrm{He}$ scattering in the hybrid quark-hadron model, Nucl. Phys., A518,717.

[58] EL-Gogary, M.M.H., Ellithi, A. Y., Hegab, M. Kh., Mohamed, A. A. (2005), Elastic scattering via quark structure of nucleons, Eur. Phys. J., A 24, 137.

[59] EL-Gogary, M.M.H. (2003), Integral formula for calculating rigorously the full Glauber series of the elastic scattering between two cluster nuclei, Phys. Rev., C68, 054609. 